\title{
La soberanía espacial argentina: desafíos y oportunidades para América Latina
}

Revista Latinoamericana de Economía y Sociedad Digital

Issue 2, agosto 2021

\section{Autores: Guadalupe Rey Sumay (iD}

DOI: $10.53857 /$ LAXW4836

Publicado: 25 agosto, 2021

Recibido: 21 marzo, 2021

Cita sugerida: Rey Sumay, Guadalupe (2021) "La soberanía espacial argentina: desafíos y oportunidades para América Latina" en Revista Latinoamericana de Economía y Sociedad Digital, Issue 2

Licencia: Creative Commons Atribución-NoComercial 4.0 Internacional (CC BY-NC 4.0)

Tipo: Estudio de caso

Palabras clave: cooperación, políticas, satélites, soberanía, tecnología

\section{Resumen}

En el presente escrito se procura abordar el proceso desarrollado en la Argentina respecto a su sistema satelital y el rol que toma el Plan Geoestacionario Argentino en la reivindicación de la soberanía espacial. Una vez delineados los intereses y las motivaciones del país respecto a su desarrollo tecnológico en materia espacial, se propone analizar el papel que toma la Argentina en la construcción de cooperación regional. A partir de un estudio de caso, el presente artículo analiza la construcción de la soberanía espacial argentina a partir del análisis de las políticas públicas desarrolladas e implementadas para su consecución. A partir de ello, se estudia cómo la construcción de la soberanía espacial le permitió a la Argentina colocarse a la vanguardia del desarrollo satelital en la región, y cuáles son las oportunidades y desafíos que esto presenta para la cooperación en materia espacial en América Latina. 


\section{Abstract}

In this paper, we seek to address the process that was developed in Argentina regarding its satellite system and the role that the Argentina Geostationary Plan plays in the claim of the spatial sovereignty. After outlining the interests and motivations of the country related to its technological development in the spatial area, the role that Argentina plays in the construction of the regional cooperation is analyzed. From a case study, this paper analyses the construction of the Argentine spatial sovereignty taking into account the analysis of the public policies that were developed and implemented for its deployment. On this basis, we have studied how the construction of spatial sovereignty allowed Argentina to become the leader in satellite development in the region, and the opportunities and challenges that this offers for the cooperation regarding spatial-related matters in Latin America.

\section{Resumo}

Neste artigo, procura-se abordar o processo desenvolvido na Argentina em relação ao seu sistema satelital e o papel desempenhado pelo Plano Geoestacionário Argentino na reivindicação da soberania espacial. Uma vez delineados os interesses e as motivações do país em relação ao seu desenvolvimento tecnológico em matéria espacial, propõe-se analisar o papel que a Argentina assumiu na construção da cooperação regional. A partir de um estudo de caso, este artigo analisa a construção da soberania espacial argentina a partir da análise das políticas públicas desenvolvidas e implementadas para a sua concretização. Com base nisso, estuda-se como a construção da soberania espacial permitiu à Argentina colocarse na vanguarda do desenvolvimento satelital na região, e quais são as oportunidades e desafios que isso traz para a cooperação em matéria espacial na América Latina.

\section{Introducción}

El presente artículo ${ }^{[1]}$ busca estudiar el proceso de construcción de la Argentina como "país espacial" y cómo este le permitió colocarse a la vanguardia del desarrollo satelital en la región. A partir de esto, se sostiene que la Argentina puede liderar el proceso de construcción de una agencia espacial latinoamericana. En primer lugar, se analizarán los esfuerzos que el país destina y destinó a la construcción de una soberanía espacial, y cómo esta construcción busca posicionar a la Argentina como referente de la región en materia espacial. En segundo lugar, se introducirá el estado de la cooperación regional en ciencia y tecnología durante el período 2005-2015, y en particular en el sector espacial, para esbozar los principales desafíos que presenta la construcción de una agencia regional en la materia. Por último, se reflexionará sobre el rol de la Argentina en la región y su potencial capacidad para liderar el proceso de integración a través de la Agencia Latinoamericana y Caribeña 
del Espacio.

\section{La construcción de la soberanía espacial argentina}

A diferencia de lo que ocurre en ciertos espacios terrestres (como la Antártida) y acuáticos (como el alta mar), en el espacio superior al subyacente al territorio nacional, los Estados no poseen la potestad de reclamar soberanía (UNOOSA, 2008), es decir, el espacio no puede ser sujeto del monopolio legítimo de la fuerza de un Estado. Esto se encuentra expreso en los Principios Gobernantes de las Actividades de los Estados en la Exploración y Uso del Espacio Exterior, incluyendo la Luna y otros cuerpos celestes, adoptado por la Asamblea General de las Naciones Unidas en 1966, y que cuenta a la fecha con la firma de 133 países (UNOOSA, 2020). No obstante, los principios sí contemplan al espacio exterior como patrimonio común de la humanidad, permitiendo la promoción de actividades pacíficas y la cooperación internacional en su explotación e investigación.

La fundación de las actividades en el espacio exterior encuentra sus raíces en la Guerra Fría y el concepto de "carrera espacial”. Las dinámicas geopolíticas de ese contexto histórico (Nucera, 2019) se trasladaron al espacio exterior, dando cuenta de que el acceso a este se correspondía con una proyección de poder. Aun luego de la caída de la Unión Soviética y con la aparición de nuevos actores (como empresas transnacionales dedicadas al desarrollo de tecnologías espaciales), el espacio siguió formando parte de la geopolítica (Nucera, 2019). Las actividades espaciales representan un instrumento para proyectar poder, ya sea por razones económicas, tecnológicas o incluso militares (Nucera, 2019). La posibilidad de acceder al espacio exterior otorga a los países la oportunidad de implementar planes de política industrial y tecnológica, para perseguir tanto objetivos políticos como también económicos. Sin embargo, no todos los países tienen igual acceso a los recursos que permiten la jerarquización en el sistema internacional. No es tema de este artículo analizar la o las causas que lo provocan, pero sí entender que para acceder al espacio terrestre se necesitan recursos que solo poseen quienes se encuentran tecnológicamente desarrollados. En este juego de poder, la capacidad que tiene un Estado de acceder a nuevas tecnologías, o más aún, desarrollarlas con sus propios medios, supone una jerarquización en el orden internacional que, desde el punto de vista geopolítico, puede posicionarlo en un lugar estratégico para influir en las reglas de juego.

El concepto de soberanía no está solamente anclado al territorio de un Estado y sus fronteras (Hurtado et al., 2019). La soberanía también es fruto de un sentido de identidad y comunidad (Kuah, 2009) y es también parte de un discurso político. Para países en desarrollo, como lo es la Argentina, el impulso de sectores económicos ligados a países desarrollados incide en el sentido de identidad y comunidad. En el plano espacial, apostar al avance tecnológico para proyectar poder y posicionarse en un campo de juego dominado por un selecto club de países supone reforzar en la comunidad el concepto de una soberanía 
espacial y tecnológica. Una posición orbital para ser ocupada por un satélite de telecomunicaciones tiene un valor estratégico, y puede ser entendida como la proyección de la soberanía territorial en el espacio (Hurtado, 2017) cuando un país posiciona un satélite propio en ella. Esta posición orbital habilita la posibilidad de hacer negocios; de participar en un mercado en crecimiento que otorga beneficios no solo económicos sino también sociales, dado el papel que juegan las comunicaciones hoy en día en cualquier actividad del ser humano. Asimismo, la adjudicación de una posición orbital también permite afianzar la soberanía tecnológica: desde el Estado puede planificarse una política de inversión en I+D que se materialice en científicos capacitados y en oportunidades competitivas para las empresas tecnológicas del país. En resumen, el acceso al espacio exterior es estratégico porque significa tener un papel en la geopolítica de las relaciones internacionales, y la presencia del Estado en su gestión resulta necesaria para la construcción de la soberanía espacial.

Ahora bien, para entender la evolución de la política espacial argentina es necesario retomar la historia de la adjudicación de las posiciones orbitales. El derrotero histórico permitirá entender por qué la Argentina a partir de 2005 decidió depositar la política espacial en manos del Estado para apostar a la soberanía espacial.

La posición orbital $72^{\circ}$ Oeste fue concedida a la Argentina por la Unión Internacional de Telecomunicaciones (UIT) en 1985. El país debía desarrollar un plan satelital que defendiera y utilizara dicha posición, ya que la no ocupación en tiempo y forma del espacio y frecuencia adquiridos a través de la UIT habilita la desestimación de la posición orbital permitiendo su uso por parte de otros. El gobierno nacional argumentó que el Estado se encontraba imposibilitado de encarar por su propia cuenta y riesgo la instalación y puesta en funcionamiento de un sistema de satélite nacional, lo que devino en librar a la inversión privada el emprendimiento ${ }^{[2]}$. La posición orbital $72^{\circ} \mathrm{O}$ fue así ocupada ${ }^{[3]}$ por Nahuelsat (una unión de empresas en su mayoría de capital extranjeros) con la puesta en órbita del satélite Nahuel 1A, construido en el exterior. Un año más tarde, en 1998, el ex presidente Carlos Menem firmó con Estados Unidos un acuerdo de reciprocidad que proponía ceder la posición Orbital $81^{\circ} \mathrm{O}$ a la Argentina, a cambio de permitir la penetración de los servicios de la empresa DirecTV en el país (Hurtado, 2017). La posición adquirida permitía el acceso a un mercado de clientes mucho mayor que la posición entonces ocupada por Nahuel 1A dado que permitía la iluminación de todo el continente americano. Para poder garantizar el servicio y disponer la ocupación de la posición orbital, la empresa Nahuelsat se ofreció a construir el segundo satélite geoestacionario, Nahuel 1B. Sin embargo, el compromiso de ocupación de la posición orbital no fue garantizado ${ }^{[4]}$, y varios países a la espera de la caducidad del derecho adquirido por Argentina comenzaron a presionar a la UIT para la desestimación de la posición orbital; entre los primeros, el Reino Unido ${ }^{[5]}$. Ante la presión extranjera, el gobierno nacional debió gestionar ante la UIT la prórroga de la asignación de la posición orbital $81^{\circ} \mathrm{O}^{[6]}$. Así, Argentina disponía tres años de gracia para la colocación de un satélite en dicha órbita. En agosto de 2004, a través de la Resolución $N^{\circ} 188$, la 
Secretaría de Comunicaciones revocó por razones de ilegitimidad ${ }^{[7]}$ la resolución que asignó el uso de la posición orbital $81^{\circ} \mathrm{O}$ a Nahuelsat, marcando los inicios de la construcción de soberanía espacial en manos del Estado Nacional. Finalmente, Nahuelsat entró en quiebra (Hurtado, 2019), poniendo en riesgo los derechos argentinos sobre las posiciones adquiridas y hasta entonces gestionadas por capitales extranjeros.

Ante el riesgo de pérdida de las posiciones orbitales asignadas a la Argentina ${ }^{[8]}$, en 2006 el ex presidente Néstor Kirchner sancionó la Ley 26.092 que crea la empresa estatal de telecomunicaciones ARSAT. Se decretó como objetivo de la empresa que instrumentara una nueva política satelital que contemplase el diseño, desarrollo, construcción en el país, lanzamiento y/o la puesta en servicios de satélites geoestacionarios en sus posiciones orbitales $^{[9]}$. Además de consolidar la soberanía en estas, ARSAT colaboraría en incrementar las capacidades del país en materia de telecomunicaciones y en permitir el desarrollo e impulso de la industria espacial argentina. La empresa también tiene a cargo la Red Federal de Fibra Óptica (que cuenta actualmente con una extensión de $31.529 \mathrm{~km}$ de fibra iluminada), la gestión del Centro Nacional de Datos ${ }^{[10]}$ y la plataforma de la Televisión Digital Abierta ${ }^{[11]}$; todos proyectos de telecomunicaciones con impacto económico en el país y con alcance federal, lo que significa generar empleo, conceder acceso a las comunicaciones y fomentar el desarrollo económico en todo el país.

El fin de la vida útil del Nahuel 1B llevó a ARSAT a migrar a los usuarios al satélite AMC-6 de la empresa internacional SES (Convergencia Latina, 2007) y a ocupar la órbita $81^{\circ}$ con satélites alquilados (Facultad de Ingeniería del Ejército, 2019). La dependencia del capital extranjero para la provisión de servicios y protección de las órbitas asignadas a la Argentina sin dudas amenazaban la construcción de una política espacial nacional, minando la soberanía argentina en el espacio ultraterrestre y postergando el posicionamiento de la Argentina entre los países con mejores tecnologías de acceso al espacio. ARSAT firmó contrato (Kirchner, 2007) para que la empresa argentina INVAP S.E. (desarrolladora hasta entonces de los satélites de observación argentinos) fuere la contratista principal (INVAP, 2021) para el desarrollo de la plataforma satelital geoestacionaria del país.

Finalmente, el 16 de octubre de 2014, Argentina posicionó en la órbita $72^{\circ}$ O su primer satélite geoestacionario nacional ARSAT-1; convirtiéndose así en el primer país latinoamericano en tener en órbita un satélite geoestacional totalmente propio (Casa Rosada, s. f.). Un año más tarde, en septiembre de 2015, se posicionó al ARSAT-2 en la órbita $81^{\circ} \mathrm{O}$, reivindicando de este modo su soberanía espacial. Ambos satélites se convirtieron rápidamente en un hito histórico del país, eliminando la dependencia de capitales extranjeros para la defensa de las posiciones orbitales y proyectando así una posición tecnológica dominante en la región. 


\section{El Plan Satelital Geoestacionario}

La puesta en valor de una política espacial en materia de telecomunicaciones se materializó poco antes del lanzamiento del ARSAT-2 a través de la publicación del Plan Satelital Geoestacionario Argentino 2015-2035, convertido en la Ley $N^{\circ} 27.208$. Entre otras cuestiones, el plan establece como objetivo fundamental la mejora en la relación potencia a bordo y peso del satélite para orientarlo hacia los estándares adoptados internacionalmente a través de sistemas de propulsión eléctricos o híbridos. El plan satelital contempla la construcción de seis satélites (teniendo en la mira el reemplazo del ARSAT-1 y ARSAT-2), y un cronograma donde se estimó la puesta en órbita del ARSAT-3 para el año 2019. Sin embargo, con la asunción de Mauricio Macri al gobierno nacional en 2015, el Plan Geoestacionario se descontinuó. A partir de entonces, comenzó un período de desfinanciamiento de ARSAT, baja inversión en ciencia y tecnología, y retrasos en los pagos a INVAP (Latam Satelital, 2018). La falta de inversión y el descuido de la política espacial pusieron en grandes aprietos los avances tecnológicos de la Argentina en materia de telecomunicaciones, sobre todo en un mercado (de satélites pequeños y medianos) donde existen pocos participantes ${ }^{[12]}$ y en donde la inserción de ARSAT le garantiza a la Argentina una posición dominante en la región.

A pocos días de haber asumido la presidencia Alberto Fernández, el 4 de enero de 2020 se anunció el relanzamiento del Plan Satelital Geoestacionario Argentino (Secretaría de Innovación Pública, 2020), enfocado en la reactivación de la fabricación y puesta en órbita del tercer satélite de la órbita, ahora denominado ARSAT SG-1 (segunda generación). Más tarde, el plan se complementó con el lanzamiento del Plan Conectar (cuyo objetivo principal es el de potenciar el acceso a los servicios TIC en la población), que contempla la extensión de la Red Federal de Fibra Óptica y la puesta en valor del Centro Nacional de Datos de ARSAT y de la Televisión Digital Abierta. Al ser consultado sobre el futuro del Plan Satelital, que según la Ley $\mathrm{N}^{\circ} 27.208$ debe ser revisado en períodos no superiores a tres años (lo que no sucedió durante la gestión de gobierno anterior), el vicepresidente de ARSAT, Guillermo Rus, quien cuenta con años de trayectoria en la empresa, puntualizó (Rey Sumay, 2021): “Es preciso primero actualizar el Plan, tal como indica la Ley. El Plan debería actualizarse contemplando el Plan Conectar (...) y debería reconsiderar el desarrollo de la plataforma eléctrica que remplazaba a la ARSAT-3K luego de la asociación entre INVAP y Turkish Aerospace Industries en GSATCOM para desarrollar una plataforma Small-Geo. Por último, el nuevo plan debería plantear nuevos horizontes para la ampliación de la flota de ARSAT". Asimismo, remarcó que "se debería buscar mantener el espíritu del plan en cuanto a la ampliación de la flota de ARSAT sobre la base de su actividad comercial dado que es la principal demandante de este tipo de satélites a la industria de fabricación local. También es preciso retomar la búsqueda de ampliar el componente nacional (o regional) de los satélites de comunicaciones fabricados en el país y la cooperación regional para potenciar las capacidades industriales". 
En resumen, desde el 2005 hasta el 2015 la política en materia satelital tomó un papel importante y estratégico en la agenda del gobierno, con el propósito de convertir al país en un "país espacial", que sea capaz de proyectar su soberanía en el espacio exterior y por sus propios medios, lo que daría cuenta de sus capacidades tecnológicas y el efecto multiplicador que tendría el avance tecnológico en la industria nacional. Asimismo, el nuevo gobierno reivindica el papel estratégico de la ciencia y la tecnología y la importancia de dar continuidad al Plan Satelital, aunque aún queda pendiente su actualización.

\section{La proyección regional y el rol de la Argentina}

El Plan Satelital Geoestacionario Argentino 2015-2035 no sólo contempla cuestiones de índole tecnológicas, de avances e innovación en telecomunicaciones, sino también la construcción de un área estratégica para la proyección argentina en la región ${ }^{[13]}$. Allí, el gobierno nacional reivindica la protección del derecho soberano sobre las posiciones orbitales y reafirma la búsqueda de un reposicionamiento en el sistema internacional en materia de fabricación y prestación de servicios de satélites geoestacionarios. Asimismo, el plan contempla la posibilidad de conformar una agencia espacial ${ }^{[14]}$ como modelo de integración regional que permita el acoplamiento de otros países de la región, lo que va en línea con la búsqueda de un posicionamiento regional.

Esta expresión de deseo de unificación y cooperación por parte de la Argentina dio su primer paso hacia su materialización a finales de 2020. Durante una conferencia preparada para tal fin, los gobiernos de Argentina y México firmaron un acuerdo para la creación de una Agencia Latinoamericana y Caribeña del Espacio (ALCE), cuyo objetivo esbozado es aprovechar y mejorar capacidades complementarias de los países de la región para impulsar el desarrollo tecnológico de un sector que posicionan como estratégico. Asimismo, se argumentó que la agencia permitirá el avance para cerrar brechas digitales y promover la industria tecnológica del continente, así como la formación de profesionales (Vizuete, 2020).

La cooperación científico-tecnológica podría definirse como el conjunto de actividades que, a través de diversos actores y múltiples instrumentos, implican una asociación y colaboración para la consecución de objetivos acordados mutuamente y que buscan un beneficio mutuo, en el ámbito de la investigación, el desarrollo científico-tecnológico y la innovación (López, 2017). En este sentido, se puede decir que la cooperación científicotecnológica extrapolada a un nivel internacional implica el establecimiento de objetivos estatales, encauzados en una agenda que persiga beneficios concretos y comunes, en pos del desarrollo de los países que establecen los vínculos para la cooperación.

Las variables internas, domésticas, también juegan un papel importante a la hora de evaluar la cooperación internacional; aun cuando resulta difícil la identificación de fuentes puramente domésticas para la interpretación de la política exterior (CEPAL, 1998). Una 
política nacional que establece entre sus prioridades el desarrollo científico-tecnológico de largo plazo tendrá un impacto en las decisiones en materia de política externa, sobre todo teniendo en cuenta que la tecnología es el motor clave del crecimiento económico de los países y regiones, y que el progreso tecnológico permite una producción eficiente de más y mejores bienes y servicios (Hausmann s. f.).

Durante los gobiernos de Néstor y Cristina Kirchner (2003-2015), Argentina profundizó la cooperación en materia científico-tecnológica con distintos países de la región (López, 2017), a través del Ministerio de Ciencia y Tecnología. La cooperación internacional tomó un papel central, y fue entendida como una herramienta fundamental para complementar y fortalecer las capacidades nacionales en el sector, sobre todo en áreas de vacancia estratégicas para el desarrollo nacional. En el Plan Argentina Innovadora 2020, desarrollado por el Ministerio de Ciencia y Tecnología en 2011, la cooperación internacional se orientó tanto al fortalecimiento de los procesos de cooperación regional en el marco de organismos existentes como el Mercosur y la Unasur (Ministerio de Ciencia, Tecnología e Innovación Productiva, 2013), como a la promoción de iniciativas bilaterales.

Durante el período analizado, se puede decir que la cooperación internacional en ciencia y tecnología sí se tradujo en iniciativas concretas de carácter bilateral, como la creación de centros binacionales o los programas de cooperación binacional (López, 2017). Sin embargo, no puede decirse lo mismo desde el punto de vista multilateral. Según Alejandra Kern, al analizar las apuestas de cooperación en ciencia y tecnología en espacios multilaterales como el Mercosur, la Unasur y la CELAC, existe una preeminencia de la retórica del regionalismo por sobre prácticas concretas de cooperación en ciencia y tecnología. Asimismo, en los espacios regionales donde se ha planteado una agenda de cooperación científico-tecnológica (sobre todo en el Mercosur), la necesidad de vinculación de los países de la región con los "centros mundiales" atravesó las relaciones de cooperación (Kern, 2014). Esto muestra que existe una noción de integración regional atravesada por la necesidad de integrar a los países latinoamericanos al mundo desarrollado, lo que podría dificultar la consolidación de una cooperación tecnológica netamente regional.

Al poner el foco en las telecomunicaciones, y específicamente en el mercado satelital, el escenario que se presenta es el mismo: escasa colaboración regional y predominancia de iniciativas bilaterales. Al ser consultado sobre la situación de la colaboración tecnológica regional, Guillermo Rus destacó que "en materia de comunicaciones satelitales hay escasa colaboración y proyectos conjuntos" (Rey Sumay, 2021). Según Diego Hurtado, en América Latina y el Caribe, la colaboración científico-tecnológica está más vinculada a iniciativas de las comunidades científicas de los países, pero no hay ejemplos de colaboración multilateral en lo que se podrían llamar sectores estratégicos, como lo es el sector espacial, que está considerado estratégico por la enorme cantidad de aplicaciones que ofrece, que van desde las telecomunicaciones hasta la observación de la Tierra (Luna, 2020). Por otro lado, la cooperación en materia espacial también estuvo atravesada por la necesidad de vincularse 
con países tecnológicamente desarrollados. Un ejemplo de esto se observa en el Plan Espacial Nacional, ejecutado por la Comisión Nacional de Actividades Espaciales (CONAE) de Argentina, que entre 2004 y 2015 estableció que el conjunto de la actividad espacial argentina debía realizarse en sociedad con entes espaciales de otros países (Ministerio de Relaciones Exteriores, Comercio Internacional y Culto, 2008), entre los que figura Brasil como uno de los latinoamericanos.

La necesidad de crear un organismo regional espacial se mencionó por primera vez en 2006, durante la Cumbre Espacial de las Américas desarrollada en Ecuador (Luna, 2020). Según el entonces subsecretario de Soberanía y Desarrollo Fronterizo de Ecuador, Diego Stacey, en el encuentro se establecieron las bases para constituir un organismo regional latinoamericano sobre el espacio (El Universo, 2006), pero que el tiempo de su constitución dependería del desarrollo de las siguientes conferencias. De hecho, la intención se repitió en otros eventos dedicados al sector (Luna, 2020), pero la agencia, hasta la fecha, no se materializó. La iniciativa se retomó finalmente a fines de 2020, en el marco de la CELAC, con la firma de México y Argentina de la Declaración sobre la Constitución de un Mecanismo Regional de Cooperación en el ámbito espacial, para la creación de la Agencia Espacial Latinoamericana y del Caribe (ALCE). En la declaración, se invita a los demás miembros de la comunidad a adherir. Entre los objetivos se destaca la necesidad de que este mecanismo cuente con la personalidad jurídica y capacidades necesarias para operar como catalizador de los esfuerzos regionales (Ministerio de Ciencia, Tecnología en Innovación, 2020), privilegiando el desarrollo de proyectos en materia espacial. Esto muestra la voluntad de crear un canal institucionalizado con reglas claras que permitan que la cooperación se materialice también de forma multilateral. En palabras de Hurtado de Mendoza, una agencia espacial sería el primer ejemplo en el cual América Latina demostraría que también es capaz de llevar adelante un proceso de integración regional desde la dimensión tecnológica, donde los países puedan aprovechar y transmitir sus complementariedades (Luna, 2020).

Sobre la firma de la declaración que da pie a la agencia, el canciller mexicano Marcelo Ebrard, declaró que la intención no es crear un organismo más, sino un instrumento que simboliza la voluntad y eficacia de América Latina y el Caribe para lograr sus objetivos comunes, para demostrar que es posible tener un desarrollo tecnológico propio (Luna, 2020). Esta declaración no es menor, puesto que transparenta la idea de que la región necesita desprenderse de la dependencia de los países desarrollados, en términos tecnológicos, con la necesidad de aunar esfuerzos para que el desarrollo provenga de la cooperación entre países de la región.

Una vez declarada la intención de crear una agencia espacial, surgen dos preguntas: ¿será posible su materialización?, ¿los países firmantes, Argentina y México, podrán impulsar el proceso? La primera tiene que ver con los desafíos que se presentan a la hora de pensar si será posible avanzar en las normas que regularán su funcionamiento, los mecanismos de cooperación e incluso las fuentes de financiamiento. La segunda apunta a pensar si 
Argentina y/o México pueden liderar el proceso hacia la materialización efectiva de la agencia; y aquí es en donde considero que el papel que juega la ciencia y la tecnología en la política doméstica y la trayectoria del país en la materia juegan un papel primordial.

El primer punto a considerar es que la creación de la agencia se promueve en el marco de la CELAC. Alejandra Kern sostiene que las reuniones de altas autoridades en ciencia y tecnología surgidas en este espacio dieron como resultado la manifestación de ciertos consensos y la explicitación de visiones compartidas que tomaron la forma de "declaraciones", sin intención de generar una agenda operativa (Kern, 2014). Es decir que, teniendo en consideración experiencias pasadas, las declaraciones no suelen avanzar hacia una materialización. Por otro lado, es importante remarcar la reciente salida de Brasil de la CELAC. Brasil es un país que lleva más de 40 años en la industria satelital y, al momento, es el país latinoamericano que lanzó la mayor cantidad de objetos al espacio exterior (36 objetos espaciales) (UNOOSA, s. f.), por lo que siendo un país con larga trayectoria en la materia, la no participación de Brasil en el proyecto podría frenar los avances de la agencia.

El segundo punto tiene que ver con las asimetrías que existen en la región. Si se observan las experiencias en materia espacial, en particular en materia satelital, las trayectorias históricas de los países latinoamericanos difieren ampliamente entre sí. No todos los países comenzaron el desarrollo de estas capacidades por los mismos motivos, ni en el mismo período de tiempo. Encontramos a Brasil y a México como los primeros países latinoamericanos en lanzar satélites al espacio en la década de los ochenta, de la mano del Estado, y una posterior privatización de las telecomunicaciones en pos de su desarrollo. En ambos países, la presencia del sector privado en materia satelital tiene más de 40 años. En el caso de Colombia, los proyectos satelitales se vieron truncados, ya sea por falta de interés de los gobiernos de turno o por cuestiones de financiamiento. Para suplir la desinversión satelital se optó por el despliegue de fibra óptica (Tellez, 2014). Venezuela y Bolivia comenzaron la exploración del desarrollo de satélites de telecomunicaciones muy recientemente, y en el caso venezolano, el motivo fue social (Agencia Bolivariana para Actividades Espaciales, 2021) y no comercial. Por otro lado se encuentra Argentina, que posee uno de los programas espaciales más ambiciosos y que se posiciona como el primer país latinoamericano (Casa Rosada s. f.) que puso en órbita satélites de telecomunicaciones puramente nacionales.

Al reflexionar sobre las asimetrías, la posición política de los gobiernos latinoamericanos también toma un papel importante. Al ser consultado sobre las dificultades de la colaboración tecnológica regional, Guillermo Rus destaca que "para avanzar en la integración regional en materia espacial, entiendo es una condición necesaria que la conducción política de las naciones que integran nuestra región tengan miradas similares; no sólo en lo que respecta al desarrollo tecnológico de cada uno de los países sino también sobre cuestiones de fondo de la economía, la inclusión, etcétera. El contexto político actual no parece ser ideal para avanzar en acuerdos amplios en la actividad espacial” (Rey Sumay, 2021). 
El tercer punto tiene que ver con formar un espacio de cooperación regional que incluya a todas las partes interesadas, no solo a entidades gubernamentales. Incluir en el espacio de cooperación a reguladores, tomadores de decisión, el sector privado y la sociedad en su conjunto puede ser en beneficio del desarrollo económico de la región y contribuye a fomentar la inversión en el sector. La necesidad de inversión no es un aspecto menor, y esto puede poner en juego el carácter puramente regional de una iniciativa con las magnitudes que tiene la creación de una agencia regional. Como observa Kern, si bien la cooperación intrarregional fortalece la idea de que es necesario lograr la autonomía en la región en ciencia y tecnología, el vínculo extrarregional es un medio para acceder a recursos materiales y a conocimientos que no están disponibles en la región (Kern, 2014), como puede ser la fuente de financiamiento. Asimismo, pensar en fuentes de financiamiento requiere reflexionar sobre el papel que tiene la ciencia y tecnología en la política doméstica de cada Estado que, como se esbozó anteriormente, influirá en la política exterior, y por ende en las decisiones que se tomen en materia de cooperación. Si los presupuestos a destinar para la creación de la agencia son bajos, difícilmente se podría pensar en la consecución de grandes iniciativas regionales con un fuerte impacto económico en la región. Más aún teniendo en cuenta el contexto actual de pandemia, en donde el crecimiento económico de la región se contrajo en un $6.5 \%$ en 2020, con una proyección de crecimiento de 5.2\% para 2021; insuficiente para retomar los valores de PBI al nivel de 2019 (World Bank. 2021).

Finalmente, este artículo propone reflexionar sobre el rol que puede tener Argentina en el proceso de construcción de una agenda espacial, y si puede ser capaz de liderar este proceso. Aquí es donde considero que la noción de soberanía espacial que tiene el país juega un papel importante. La búsqueda de esta soberanía se tradujo en la creación de una empresa de telecomunicaciones estatal, y en la puesta en marcha de un plan satelital ambicioso, a través del cual Argentina pudo poner en órbita dos satélites GEO 100\% nacionales, además de los referidos a aplicaciones científicas.

En la actualidad, el gobierno nacional decidió retomar el Plan Geoestacionario y comenzar la construcción de un tercer satélite. Asimismo, el gobierno del presidente Alberto Fernández decidió restituir el Ministerio de Ciencia y Tecnología (que había sido desplazado al nivel de Secretaría por la administración anterior), en clara alusión al papel estratégico que el gobierno le otorga al sector. En palabras del presidente, la ciencia y la tecnología son pilares fundamentales de la Argentina del futuro, siendo las sociedades más ricas las que han invertido en conocimiento (Periferia, 2020). Recientemente, en el aniversario número 30 de la CONAE en mayo de 2021, el ministro de Ciencia y Tecnología destacó que el desarrollo satelital y el acceso al espacio constituyen no sólo elementos de soberanía para el país sino también el impulso necesario para el desarrollo de una industria de alta tecnología; y finalmente puntualizó que se continuará impulsando la cooperación internacional como un factor esencial en el desarrollo espacial de la Argentina (Ministerio de Ciencia, Tecnología e Innovación, 2021). Dado el rol central que toma la ciencia y la tecnología en el país, y la voluntad política que ya se demostró con la firma de la 
declaración para la creación de la agencia espacial, Argentina se encuentra en una posición favorable para liderar el proceso de creación, puesto que la integración regional se presenta como estratégica para el desarrollo espacial de la Argentina.

\section{Conclusiones}

En el período analizado, la política en ciencia, tecnología e innovación tomó un rol central en la agenda del gobierno nacional. Específicamente, el desarrollo de tecnología espacial en manos del Estado significó proyectar la soberanía territorial en el espacio ultraterrestre, siendo este también un espacio donde se reproduce la geopolítica internacional. El carácter estratégico del desarrollo de la industria espacial del país quedó plasmado en el Plan Geoestacionario Argentino y en la creación de la empresa estatal ARSAT, que conforme pasaron los años, su rol en la política espacial argentina resulta central. Gracias a una política espacial planificada y apoyada desde el gobierno nacional, Argentina logró ocupar sus posiciones orbitales asignadas con dos satélites de comunicaciones 100\% nacionales, posicionándose así como el primer país latinoamericano en lograrlo. En cada uno de estos hitos, el gobierno nacional reivindicó el concepto de soberanía. Por otro lado, en el Plan Geoestacionario, Argentina contempla el impulso de la integración regional, estableciendo la posibilidad de construir una agencia espacial para aunar los esfuerzos de cooperación científicos-tecnológicos en materia espacial. Este artículo se propuso reflexionar sobre los desafíos que presenta una agencia espacial regional y el rol que puede tener Argentina en este proceso, desde su posicionamiento como país espacial. Los estudios actuales sobre cooperación científico-tecnológica en América Latina arrojan resultados no muy alentadores. La cooperación entre países latinoamericanos se concentró en iniciativas bilaterales. Los intentos por establecer las bases para la cooperación en organismos multilaterales como el Mercosur, la Unasur y la CELAC fueron escasos, y la mayoría quedaron plasmados únicamente en el discurso político. Con la firma de la declaración para la construcción de la Agencia Latinoamericana y Caribeña del Espacio, Argentina y México dieron el primer paso para la creación de un foro multilateral en la materia, aunque el mismo presenta varios desafíos: (i) las iniciativas en ciencia y tecnología el marco de la CELAC no parecen generar agendas operativas, (ii) las experiencias en materia espacial y el acceso a estas tecnologías no son simétricas entre los países de la región, lo mismo ocurre con las capacidades de financiación, (iii) las iniciativas multilaterales también requieren pensar en la inclusión de actores no gubernamentales, como empresas multinacionales y espacios de la sociedad civil y (iv) la salida de Brasil de la CELAC podría no favorecer el proceso de integración espacial en la región, teniendo en cuenta los avances de Brasil en la materia. Si bien los desafíos son múltiples, en este artículo se considera que Argentina ha dado el primer paso necesario para la integración de la región. Dado el rol central que toma la ciencia y la tecnología en el país, y la voluntad política que se demuestra desde los altos cargos de la administración actual, se concluye que Argentina se encuentra en una posición 
favorable para liderar el proceso de integración regional a través de la ALCE, puesto que esta se presenta como estratégica para el desarrollo espacial de la Argentina.

\section{Bibliografía}

- Agencia Bolivariana para Actividades Espaciales. (2021). Venesat-1 descripción e historia. Recuperado el 12 de marzo de 2021. http://www.abae.gob.ve/?page_id=1090

- Casa Rosada. (s. f.). El ARSAT-1 es una conquista de todos los argentinos. Recuperado el 7 de junio de 2021.

https://www.casarosada.gob.ar/27976-el-arsat-1-es-una-conquista-de-todos-los-argentinos-afi rmo-la-presidenta

- CEPAL, N. (1988). Análisis y formulación de la política exterior.

- Convergencia Latina. (23 de agosto de 2007). Avanza la migración del Nahuel hacia el AMC 6 de SES.

http://www.convergencialatina.com/Nota-Desarrollo/86365-3-51-Avanza_la_migracion_del_N ahuel_hacia_el_AMC_6_de_SES

- El Universo. (28 de julio de 2006). Terminó en Quito la Cumbre Espacial de las Américas. https://www.eluniverso.com/2006/07/28/0001/1064/FFDD8B89AFC6485F9A61D80F49FA8C FF.html

- Facultad de Ingeniería del Ejército. (2019). Satélites geoestacionarios, posición 81 para Argentina. https://www.fie.undef.edu.ar/ceptm/?p=3661

- Gobierno de la República Argentina. (2006). Ley N² 26.092. ARSAT.

- Gobierno de la República Argentina. (2015). Ley N²7.208. Plan Satelital Geoestacionario Argentino.

- Gobierno de la República Argentina. (2010). Decreto 1552. Plan Nacional de

Telecomunicaciones "Argentina Conectada".

- Gobierno de la República Argentina. (2010). Decreto 364. Plataforma Nacional Digital Terrestre.

- Hausmann, R. y Domínguez, J. (s. f.). Knowledge, Technology and Complexity in Economic Growth. Recuperado el 5 de junio de 2021. Harvard University.

https://rcc.harvard.edu/knowledge-technology-and-complexity-economic-growth

- Hurtado De Mendoza, D., Bianchi, M. D., \& Lawler, D. (2017). Tecnología, políticas de Estado y modelo de país: el caso ARSAT, los satélites geoestacionarios versus "los cielos 
abiertos”. Epistemología e Historia de la Ciencia, 2(1), 48-71.

- Hurtado de Mendoza, D. (8 de septiembre de 2017). Soberanía satelital [transmisión en directo]. Facebook.

https://www.facebook.com/100002315137807/videos/1466881600065664/

- Hurtado de Mendoza, D. , \& Blinder, D. (2019). Satélites, territorio y cultura: ARSAT y la geopolítica popular. Revista Transporte y Territorio, (21), 6-27.

- Hurtado, D., \& Loizou, N. (2019). Desregulación de sectores estratégicos en contexto semiperiférico: las comunicaciones satelitales en Argentina, 1991-2006. América Latina en la historia económica, 26(1).

- INVAP. (S. f.). Satélites de comunicaciones. Recuperado el 06 de junio de 2021. https://www.invap.com.ar/areas/espacial/satelites-de-comunicaciones/

- Kern, A. (2014). La agenda científica y tecnológica en los regionalismos de América Latina. En Conferencia Internacional Conjunta FLACSO-ISA (Vol. 23, pp. 1-22).

- Kuah, A. W. (2009). Sovereignty and the politics of identity in international relations.

- Kirchner, N. (2007). Mensaje del Presidente Néstor Kirchner a la Asamblea Legislativa en el inicio del 125 período de sesiones ordinarias del Congreso de la Nación. Recuperado el 6 de junio de 2021.

http://www.cfkargentina.com/nestor-kirchner-en-la-apertura-de-sesiones-del-congreso-2007

- Latam Satelital. (1 de diciembre de 2018). Informe de INVAP sobre contratos con el Estado Nacional. http://latamsatelital.com/informe-invap-contratos-estado-nacional

- López, M. P. (2017). La cooperación en ciencia y tecnología entre Argentina y los países de América Latina. El caso del Ministerio de Ciencia, Tecnología e Innovación Productiva (2007-2015).

- Luna, Nadia. (15 de octubre de 2020). Integración espacial en América Latina. TSS UNSAM. https://www.unsam.edu.ar/tss/integracion-espacial-en-america-latina/

- Ministerio de Ciencia, Tecnología e Innovación Productiva. (2013). Argentina Innovadora 2020: Plan Nacional de Ciencia, Tecnología e Innovación. Lineamientos estratégicos 2012-2015.

- Ministerio de Ciencia, Tecnología e Innovación. (9 de octubre de 2020). Argentina y México dan el primer paso para la creación de la Agencia Espacial Regional de América Latina y el Caribe.

https://www.argentina.gob.ar/noticias/argentina-y-mexico-dan-primer-paso-para-la-creacionde-la-agencia-espacial-regional-de

- Ministerio de Ciencia, Tecnología e Innovación. (28 de mayo de 2021). CONAE cumple 30 
años afianzando la tecnología espacial argentina.

https://www.argentina.gob.ar/noticias/conae-cumple-30-anos-afianzando-la-tecnologia-espac ial-argentina-0

- Ministerio de Relaciones Exteriores, Comercio Internacional y Culto. (23 de mayo de 2008). La Argentina amplía la cooperación espacial con Europa.

https://www.mrec.gob.ar/es/actualidad/comunicados/la-argentina-amplia-la-cooperacion-esp acial-con-europa

- Nucera, G. G. (2019). International Geopolitics and Space Regulation. Oxford Research Encyclopedia of Planetary Science.

- Periferia. (26 de febrero de 2020). Alberto Fernández reivindicó a la ciencia y la tecnología como "pilares fundamentales" de su gestión.

http://www.periferiaciencia.com.ar/noticia.php?n=737

- Rey Sumay, G. (11 de junio de 2021). Entrevista a Guillermo Rus.

- Secretaría de Comunicaciones, Gobierno de la República Argentina. (2004). Resolución 188. https://www.argentina.gob.ar/normativa/nacional/resoluci\%C3\%B3n-188-2004-97569

- Secretaría de Comunicaciones, Gobierno de la República Argentina. (1998). Resolución 2593. https://www.argentina.gob.ar/normativa/nacional/resoluci\%C3\%B3n-2593-1998-54656

- Secretaría de Innovación Pública. (2020). Relanzamiento del plan satelital geoestacionario. https://www.argentina.gob.ar/noticias/relanzamiento-del-plan-satelital-geoestacionario

- Téllez García, L. (2014). Satélites de telecomunicaciones en Colombia. Pasado, presente y futuro (Bachelor's thesis, Bogotá-Uniandes).

- United Nations Office for Outer Space Affairs. (s. f. ). Online Index of Objects Launched into Outer Space. Recuperado el 11 de junio de 2021 de

https://www.unoosa.org/oosa/osoindex/search-ng.jspx?lf_id=

- United Nations Office for Outer Space Affairs. (2008). United Nations Treaties and Principles on Outer Space and Related General Assembly Resolutions. https://www.unoosa.org/pdf/publications/st_space_11rev2E.pdf.

- United Nations Office for Outer Space Affairs. (2020). Status of International Agreements Relating to Activities in Outer Space.

https://www.unoosa.org/documents/pdf/spacelaw/treatystatus/TreatiesStatus-2020E.pdf

- Vizuete, D. (12 de octubre de 2020). ¿América Latina y el Caribe en las puertas de la carrera espacial internacional?. Periferia.

http://www.periferiaciencia.com.ar/noticia.php?n=1496

- World Bank. (2021). Global economic prospects, June 2021. 
Bio: Guadalupe Rey Sumay es Licenciada en Estudios Internacionales de la Universidad Torcuato Di Tella (Argentina) en donde ejerció la docencia de la cátedra Comercio Internacional, como profesora auxiliar. Actualmente se desempeña como Especialista de Public Policy, Risk \&amp; Strategy para el sector de Tecnología y Telecomunicaciones en Cefeidas Group International Advisory Firm. Previamente, trabajó como asesora en el Ministerio de Economía de la Ciudad de Buenos Aires. Es Becaria de la $12^{\circ}$ edición de la Escuela del Sur de Gobernanza de Internet y cuenta con un curso de posgrado en Oportunidades y Desafíos para profesionales TIC de la Universidad Nacional de Avellaneda. Formó parte del Grupo de Jóvenes Investigadores del Instituto de Relaciones Internacionales de la Universidad Nacional de La Plata, donde publicó artículos de análisis de coyuntura internacional. Guadalupe posee un nivel avanzado de inglés, francés y ruso.

\section{Notas}

Este artículo fue escrito a título personal y representa una opinión individual, y no debe interpretarse como la opinión de ninguna institución a la que esté o haya estado vinculada en mi vida profesional y académica.

$\uparrow 2$ Resolución 2593, 27 de noviembre de 1998, Secretaría de Comunicaciones.

$\uparrow 3$ Resolución 2593, Secretaría de Comunicaciones.

$\uparrow 4$ Ley N 27.208. Plan Satelital Geoestacionario Argentino 2015-2035, 2015: 10.

$\underset{\uparrow \mathbf{1}}{\uparrow \mathbf{5}}$, Ley N²7.208. Plan Satelital Geoestacionario Argentino: 10.

†6 Ley $N^{\circ}$ 27.208. Plan Satelital Geoestacionario Argentino: 13.

17 Resolución 188, 17 de agosto 2004, Secretaría de Comunicaciones.

$\uparrow 9$ Ley $\mathrm{N}^{\circ} 26.092,5$ de abril de 2006, Congreso Nacional.

†10 Decreto 1552, 21 de octubre de 2010, Poder Ejecutivo Nacional. 
$\uparrow 11$ Decreto 364, 15 de marzo de 2010, Poder Ejecutivo Nacional.

$\uparrow 12$ Ley N² 27.208. Plan Satelital Geoestacionario Argentino: 53.

$\uparrow 13$ Ley N² 27.208. Plan Satelital Geoestacionario Argentino: 57.

$\uparrow 14$ Ley $N^{\circ}$ 27.208. Plan Satelital Geoestacionario Argentino: 58. 